\title{
"Study And Assessment Of Drug-Drug Interactions In Hospitalised Patients In Quaternary Care Hospital"
}

\author{
"Bhavana Chowdary $\mathrm{M}^{1}$, Chaitra.T ${ }^{1}$, D Sai Muktha Sushma ${ }^{1}$, \\ Johncy Nathasha ${ }^{1}, \mathrm{R}$ Srinivasan ${ }^{2}$ \\ (Dept. of clinical pharmacy practice, PES College of pharmacy affiliated to RGUHS, INDIA) \\ Corresponding Author: Bhavana Chowdary $M^{1}$
}

\begin{abstract}
Drug-Drug Interactions (DDI) in patients receiving multi-drug therapy are of wide spread. Such interactions are one of the important cause of adverse drug reactions (ADRs) and may lead to increased risk of mortality and morbidity. Hence, health cost will increase. This study estimates to find out the potential DDIs among the hospitalized patients. The final results of the study concluded with a major preponderance towards ATORVASTATIN and ASPIRIN In which the interaction of ATORVASTATIN and PANTOPRAZOLE can lead to (insert what will happen if HMG coa increases) and interaction between ASPIRIN and HEPARIN can lead to (insert what will happen if the interactions occur) further intervention by clinical pharmacists to reduce such adversities have to be implemented.
\end{abstract}

Keywords: drug-drug interaction, adverse drug reaction, patient medical record.

\section{Introduction}

An interaction is said to occur when the effects of one drug are changed by the presence of another drug, herbal medicine, and food drink or by some environmental chemical agent. Drug-drug interaction is a modification of the effect of a drug when administered with another drug. The effect may be an increase or decrease in the action of either substance, or it may be an adverse effect that is not normally associated with either drug. This has become an important issue in the health care. ${ }^{1}$

The majority of the patients are treated with more than one drug simultaneously. Reasons for the treatment with multiple drugs include the treatment of multiple ailments in the same patient and the use of multiple drugs for the same ailment. With the increasing median age of the population, and the now known effectiveness of multiple therapy regimens for viral diseases, cancer, cardiovascular diseases and infectious diseases, so exposure of a patient to multiple drugs is a common rather than occurrence. ${ }^{2}$

The outcome can be harmful if the interaction causes an increase in the toxicity of the drug. For example, there is a considerable increase in risk of severe muscle damage if patients on statins start taking azole antifungals. A reduction in efficacy due to an interaction can sometimes be just as harmful as an increase: patients taking warfarin who are given rifampicin need more warfarin to maintain adequate and protective anticoagulation. These unwanted and unsought-for interactions are adverse and undesirable but there are other interactions that can be beneficial and valuable, such as the deliberate co-prescription of antihypertensive drugs and diuretics in order to achieve antihypertensive effects possibly not obtainable with either drug alone. The mechanisms of both types of interaction, whether adverse or beneficial, are often very similar. ${ }^{1}$

The basic and clinical scientific issues underlying pharmacokinetic drug interactions are becoming increasingly complex as poly pharmacy becomes more common and more drugs with enzyme-inducing or inhibiting properties are introduced into clinical practice. A well-planned, integrated approach is needed to address the clinical problems. Ideally, the approach should incorporate the collaborative participation of individuals with expertise in molecular pharmacology, cytochrome biochemistry, in vitro metabolism, clinical pharmacokinetics-pharmacodynamics, and clinical therapeutics. The ultimate goal should be the informed and safe use of drug combinations in clinical practice. Drug interactions are said to occur when the pharmacological activity of a drug is altered by the concomitant use of another drug or by any pharmacologically active substance. The drug whose activity is affected is affected by such an interaction is called as object drug and the agent which precipitates such an interaction is referred to as the precipitant. The net effect of a drug interaction is Increased or decreased effect (quantitative) Rapid or slow effect (seldom qualitative) Precipitation of newer or increased adverse effects. Most interactions are undesirable. Rarely desirable (beneficial) example:enhancement of activity of penicillin's when administered with probenecid. ${ }^{3}$ 


\section{Methodology}

Data was retrieved from patient case file and Patient Medical Record (PMR) and stored in a data collection form for a period of 6 months. DDIs were identified using Lexi-Comp, database system. The drugdrug interactions were classified as major, moderate and minor. Inclusion criteria were only Hospitalized patients and Exclusion cretiria were Prescription containing less than three drugs.

\subsection{DATA COLLECTION FORM:}

The data collection form was developed by referring available literatures. It includes patient demographics, laboratory results, final diagnosis and medication chart.

\subsection{DOCUMENTATION:}

The data collected from the patients was documented for further analysis.

Microsoft excel software was used for statistical analysis.

\section{Results}

This was a prospective observational study conducted for 6 months.During the study total of 202 prescriptions were collected and analysed.Out of 202 prescriptions 131 were male (64.8\%),71 were females(35.1\%).

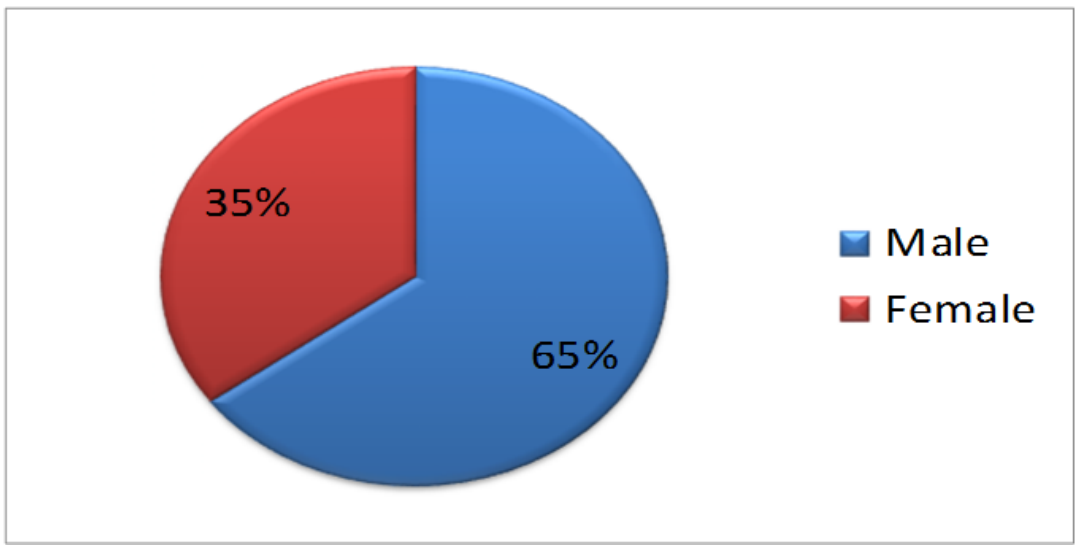

Fig .1: Grouping of patient based on gender

Out of 202 prescriptions, 12 prescriptions were of age <20years(6\%),35 were between 2035 years(17\%),34 prescriptions were between 36-50years(17\%),68 prescription were between 5165 years $(34 \%), 47$ prescription were between $66-80$ years $(23 \%), 6$ prescription were>80years( $3 \mathrm{ss} \%)$.

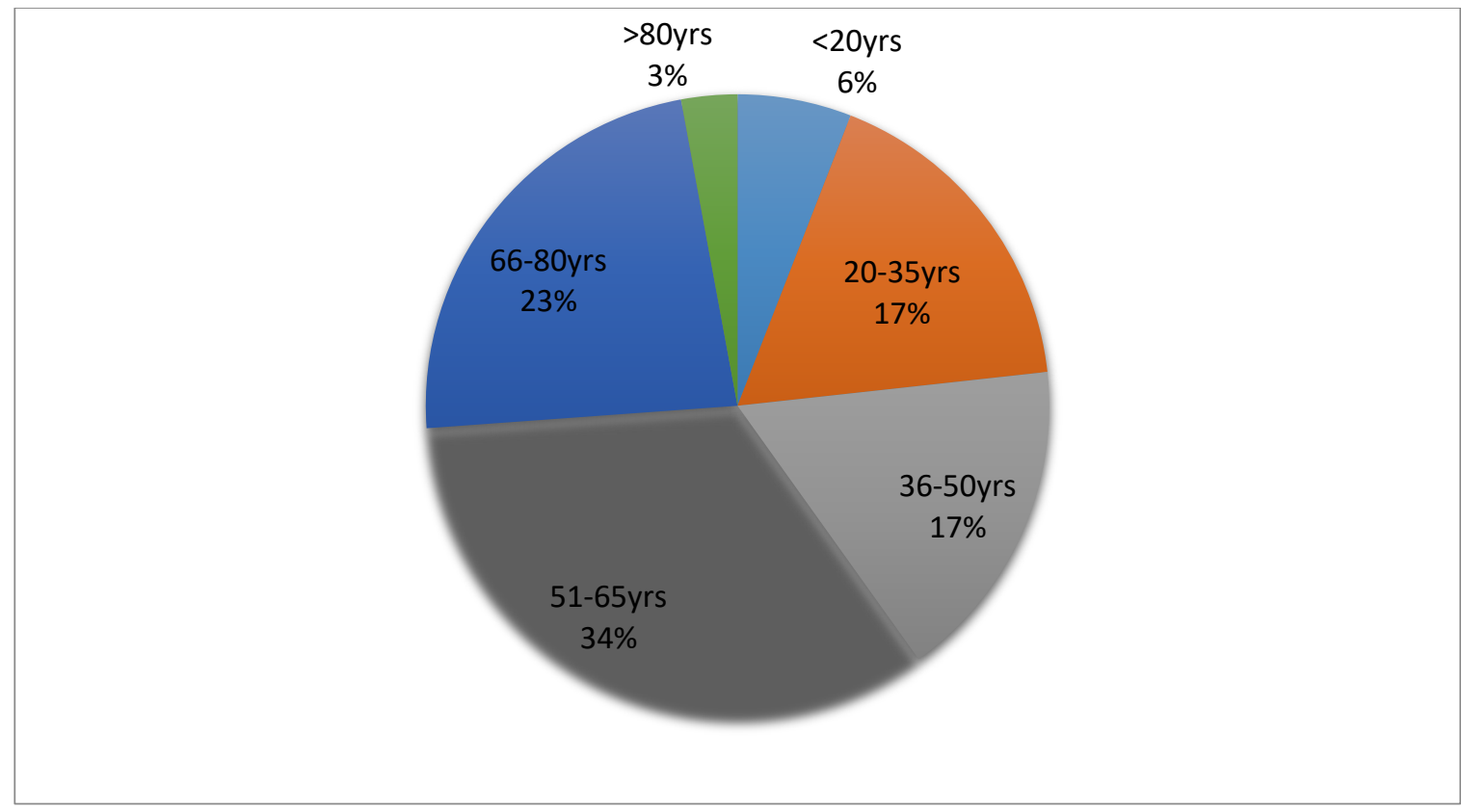

Fig.2: Grouping of patient based on age.

Out of 202 prescriptions, 181(10.3\%) prescriptions were identified with DDIs and 21(89.6\%) were without DDIs. 
Table 1 : Prevalence of DDIs in study population

\begin{tabular}{|l|l|l|}
\hline Type of case & Number of prescriptions & Percentage \\
\hline Cases with DDIs & 181 & 10.3 \\
\hline Cases with out DDIs & 21 & 89.6 \\
\hline Total No. of cases & 202 & 100 \\
\hline
\end{tabular}

The identified DDIs were analysed and classified based on severity as major, moderate and minor.A total of 1238 DDIs were identified of which 583 combinations were obtained from 181 prescription. From these $314(25.36 \%)$ were of major, $821(66.31 \%)$ were of moderate and $103(8.31 \%)$ were of minor.

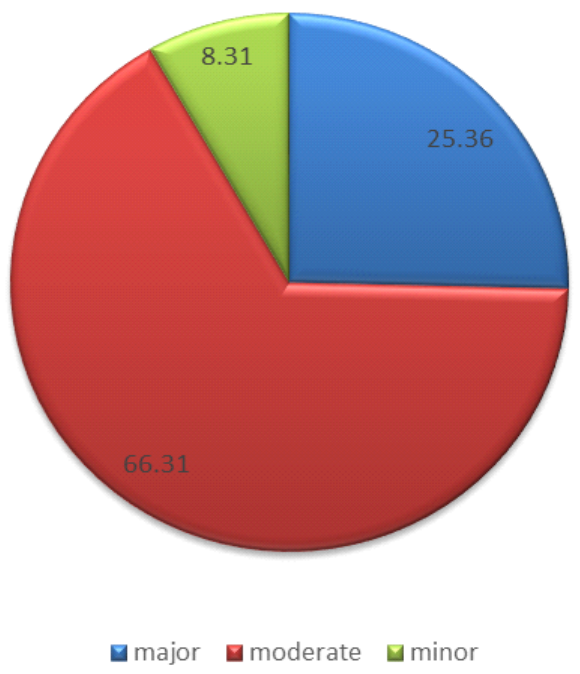

Fig.3: Distribution of DDIs according to severity.

The drugs are classified according to the Lexi-Comp,Inc. Verion: 2.7.5 Copyright 2016, LexiComp,Inc. database where the drugs are classified as $\mathrm{X}$ (avoid combiation), $\mathrm{D}$ (consider therapy modification), $\mathrm{C}$ (monitor therapy), $\mathrm{B}$ (no action needed), A(no known interaction).Out of 1238 DDIs, 53 were $\mathrm{X}(4.28 \%), 177$ were $\mathrm{D}(14.29 \%), 826$ were $\mathrm{C}(66.72 \%), 166$ were $\mathrm{B}(13.40 \%), 16$ were $\mathrm{A}(1.29 \%)$.

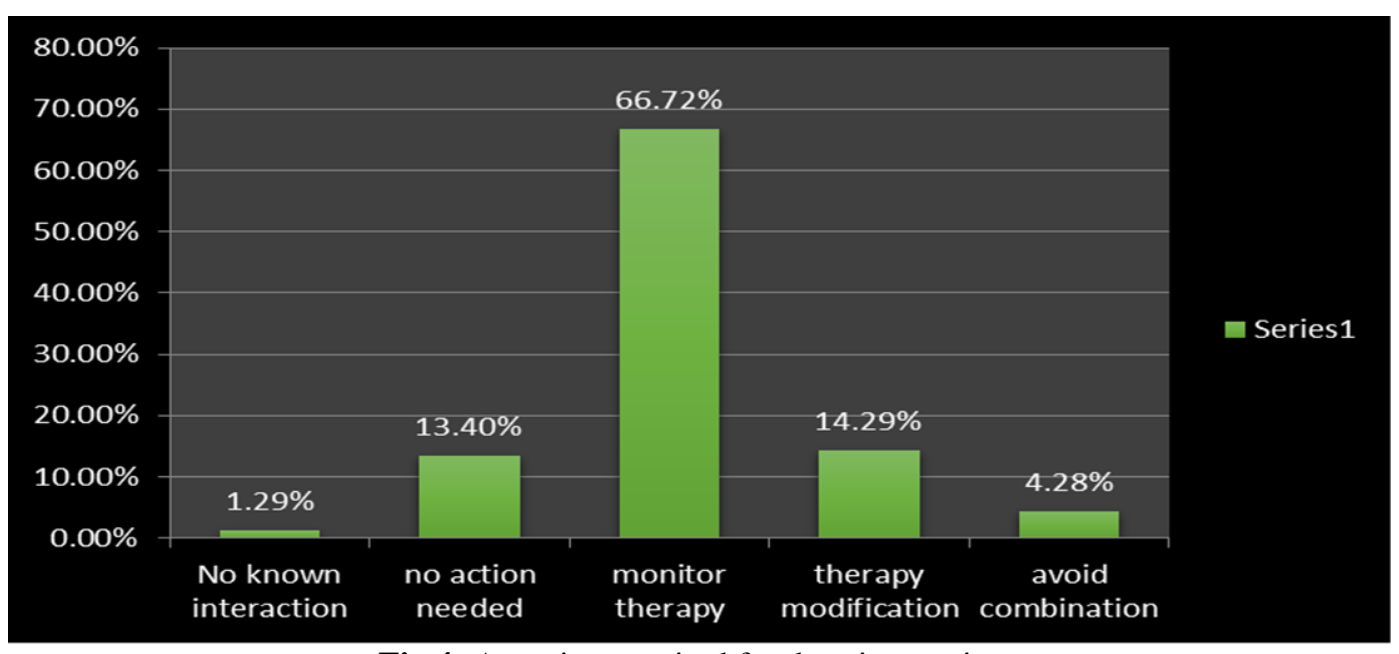

Fig.4: Attention required for drug interactions

The most prevalent DDIs were identified. The most frequent interacting combinations were Atorvastatin+ pantoprazole, clopidogrel+aspirin, clopidogrel+atorvastatin, aspirin+heparin, ondansetron+tramadol. 


\section{Discussion}

As per the evidence obtained there are a vast spread of reasons contributing to the drug interactions some of them are discussed as follows

\subsection{RISK FACTORS CONTRIBUTING TO DRUG INTERACTIONS:}

Multiple drug therapy: This is very common in most acute and chronic care settings, for e.g., therapy in patient suffering from hypertension and congestive heart failure includes antihypertensives as well as digitalis which together may lead to abnormal heart rhythms. Concurrent use of non-prescription drugs, for e.g. aspirin as well as herbal medications also lead to drug interactions. Theoretically, the possibility for drug interactions to occur is over $50 \%$ when a patient is receiving five medications, and the probability increases to $100 \%$ when seven drugs are used.

Multiple prescribers: some individuals go to more than one physician, and it is common for a patient to be treated by one more specialist in addition to a family doctor. Eg: one doctor may prescribe an anxiolytic for a patient while another prescribes an antihistamine having sedative properties with a possible consequence of an excessive depressant effect.

Multiple diseases: some patient take several drugs owing to their suffering from more than one disease e.g. a patient with both diabetes and hypertension takes hypoglycemics and beta blockers which result in decreased response to anti-diabetic drug resulting in elevated blood sugar level. Advanced age of patients: increased tendency of drug interactions in elderly due to decrease in liver function. Drug related factors: clinically significant interactions are most likely to occur between drugs that have potent effects and narrow therapeutic index. Poor patient compliance: multiple drugs, inadequate information by pharmacist or doctor, confusion regarding taking medicines which may lead to under dosing or overdosing and consequent drug interactions.

In this study total of 202 prescriptions were collected. According to gender classification there were $131(64.8 \%)$ males and $71(35.1 \%)$ females. Then according to age classification out of 202 prescriptions ,12 prescriptions were of age <20years(5.9\%),35 prescriptions were between 20-35years(17.32\%), 34 prescriptions were between 36-50years $(16.38 \%)$,68 prescriptions were between 51-65years(33.6) prescriptions were between $66-80$ years $(23.2 \%)$ and 6 prescriptions were $>80$ years(2.9\%). Based on severity out of 202 prescriptions, 181(10.3\%) prescriptions were identified with DDIs and 21(89.6\%) were without DDIs. From these total 181 DDIs, 314(25.36\%) were major, 821(66.31\%) were of moderate and 103(8.31\%) were of minor. A total of 1238 DDIs were identified of which 583 different combinations were obtained from 181 prescriptions.

The drugs are classified according to the Lexi-Comp,IncVersion: 2.7.5 Copyright 2016 database where the drugs are classified as $\mathrm{X}$ (avoid combination), $\mathrm{D}$ (consider therapy modification), $\mathrm{C}$ (monitor therapy), $\mathrm{B}$ (no action needed), $\mathrm{A}$ (no known interaction). Out of 1238 DDIs, 53 were $\mathrm{X}(4.28 \%), 177$ were $\mathrm{D}(14.29 \%), 826$ were $\mathrm{C}(66.72 \%), 166$ were $\mathrm{B}(13.40 \%), 16$ were $\mathrm{A}(1.29 \%)$.

Classification based on number of drugs prescribed, out of 202 prescriptions 39 patients were prescribed with 3-6 drugs in which 71 DDIs were found (5.46\%), 90 patients were prescribed with 7-10 drugs in which 431 DDIs were identified (33.17\%), 41 patients were prescribed 11-14 drugs in which 363 DDIs were identified (27.94\%), 26 patients were prescribed with 15-18 drugs in which 346 DDIs were identified (26.6\%), 6 patients were prescribed with greater than 19 drugs in which 88 DDIs were identified (6.77\%). The most frequent interacting combinations were Atorvastatin+ Pantoprazole, Clopidogrel+ Aspirin, Clopidogrel+ Atorvastatin , Aspirin+ Heparin , Ondansetron+ tramadol. Similar study was conducted by O Jimmy Devi, Blessy KG, Hephzibah MC, Vinay DM, Sushilkumar PL at tertiary care Teaching Hospital, Davangere. It also shows that the most prevalent DDI are with Aspirin+Clopidogrel.

\section{Conclusion}

The Drug-Drug Interactions are frequent among the hospitalized patients who were prescribed with more than three drugs. The drugs that are significantly found having major preponderance are "ATORVASTATIN+PANTOPRAZOLE and ASPIRIN+HEPARIN". Prevalence of drug interactions increases by a linear mode according to numbers of drugs prescribed, number of therapeutic drug classes, patient's gender and age. The results were obtained according to the Theoretical hypothesis obtained by us in hospitalised set up. So these interactions has been monitored by us to minimize the potential adverse drug reactions and intervention as appropriate.

\section{Acknowledgements}

We express our sincere gratitude to all those who have been associated with this project and have helped us with it and made it a worthwhile experience. Our greatest regards to the ALMIGHTY for bestowing upon the courage to face the complexities of life and to complete this project. We wish to express our sincere gratitude to our teacher and guide Mr. R. SRINIVASAN, Head of Pharmacy Practice Department, PES College 
of pharmacy, Bengaluru. His wide knowledge and his logical way of thinking have been of great value for us. His understanding, encouraging and personal guidance have provided a good basis for the present thesis. We would also like to thank Mrs. Apurva Dev, Dr. Sanjay Sharma, Dr.PonnamPravalika and all other teaching and nonteaching staffs whose constant guidance helped us completing our project. We express our sincere thanks to our classmates and our senior ChinnuReeba George, Priya Thomas and Akshata.N and junior Navya Daniel for their constant supporting. Our deepest gratitude goes to our parents, brother especially Samji john Kumar and cousin Parthiben and friends Phalgun, kartheek for their unflagging love and support throughout our life.

\section{References}

[1] Karen Baxter, SMildred Davis, Samuel Driver, Chloë SAJ Hatwal, MResC Rhoda Lee, Alison Marshall et al. stockley’s drug interactions, $8^{\text {th }}$ Ed. USA : pharmaceutical press. 2008; 1.

[2] AlbertP.Li, J. Thomas August, M. W. Anders, Ferid Murad, Joseph T. Coyle, drug-drug interactions scientific and regulatory perspectives. Volume 43. USA: Academic press. 1997; 1.

[3] D.M. Brahmankar, Sunil B jaiswal. Biopharmaceutics and pharmacokinetics a treatise, $2^{\text {nd }}$ Ed. Nagpur: M K Jain for Vallabh Prakash. 2009; 224-233.

[4] Nabovati E, Vakili-Arki S, Taherzadeh Z, Hasibian M, Hanna A, Eslami S. Drug-drug interactions in inpatient and outpatient settings in Iran: a systematic review of the literature. DARU journal of pharmaceutical sciences. 2014; 22:52.

[5] Jurlink DN, Mamadani M, Laupacis A, Redelmeier DA. Drug-drug interactions among elderly patients hospitalized for drug toxicity. Journal of American medical research. 2003; 209(13):1032-1038

[6] Kasyap M, D'CRUZ S, Sachdev A, Tiwari P. Drug-drug interactions and their predictors: Results from Indian elderly inpatients.Pharmacy Practice. 2013; 11(4):191-195.

[7] Patel LD, Acharya LD, Rajakannan T, Surulivelrajan M, Vasudeva. Potential drug interactions in patients admitted to cardiology wards of a southIndian teaching hospital. Australasian Medical Journal. 2011; 4(1): 9-14.

[8] Reis AD, Cassiani S. Prevalence of potential drug interactions in patients in an intensive care unit of a university hospital in Brazil. CLINICS. 2011; 66(1):9-15

[9] Wisniowska B, Tylukti Z, Wyszogrodzka G, Polak S. Drug-drug interactions and QT prolongation as a commonly assessed cardiac effect - comprehensive overview of clinical trials. BMC Phrmacology and toxicology. 2016;17:12.

[10] Lubinga SJ, Uwiduhaya E. Potential drug-drug interactions on inpatient medication prescriptions at MRRH in western Uganda:prevalence,clinical importance and associated factors. African health science. 2011;11(3): 499-507.

[11] Barot.PA, Malhotra SD, Patel VJ. Evaluation of potential drug-drug interactions in patients at emergency medicine department at tertiary hospital: prospective study. International journal of scientific study. $2015 ; 3: 48-53$.

[12] Kapadia J, DhavalT,Chetna D, Ramkumar D.A study of potential drug drug interactions in indoor patients of medicine department at a tertiary care hospital. Journal of Pharmaceutical science. 2013; Volume 3[10]:89-96

[13] Mateti UV, Rajakannan T, Nekkanti H, Rajesh V, Mallaysamy SR, Ramachandran P. Drug-drug interactions in Hospitalized Cardiac Patient. Journal of Young Pharmacists. 2011; 3:329-33.

[14] Rama M, Viswanathan G, Acharya LD, Attur RP, Reddy RP, Raghavan S. Assessment of Drug-Drug interactions among renal failure patients of nephrology ward in a south Indian tertiary care hospital. Indian J. Pharm. Sci. 2012; 74 (1): 63-68.

[15] S.Ray, J. Pramanik, M. Bhattacharyya, S.Todi. Prospective observational evaluation of incidences and implications of Drug-Drug interactions induced adverse drug reactions in critically ill patients. Indian Journal of Pharmaceutical Sciences. $2010 ; 72$ (6):787792 .

[16] Kohler. Drug-drug interactions in medical patients: effects of in hospital treatment and in relation to multiple drug use. Journal of clinical pharmacology and therapeutics. 2000; 38 (11):504-513.

[17] Devi O, Blessy KG, Hephzibah MC, Vinay DM, Sushilkumar PL. To Identify and Analyze Potential Drug- Drug Interactions from the Medication Charts of Medicine Units of a Tertiary Care Teaching Hospital. International Journal for Pharmaceutical Research Scholars. 2015; 4:196-204.

[18] Sankar V, Saaed Y, Joseph RM, Azizi H, Thomas PM. Serious drug-drug interactions in the prescription of diabetic patients. Journal of medical sciences. 2015; 3(4): 93-103.

[19] Lexi-Comp,Inc. VERSION;2.7.5 Copyright 2016, Lexi-Comp, Inc.

IOSR Journal of Pharmacy and Biological Sciences (IOSR-JPBS) is UGC approved Journal with Sl. No. 5012, Journal no. 49063.

Bhavana Chowdary M. "“Study And Assessment Of Drug-Drug Interactions In Hospitalised Patients In Quaternary Care Hospital"." IOSR Journal of Pharmacy and Biological Sciences (IOSR-JPBS) 12.4 (2017): 71-75. 